\title{
Phase Transitions of GUP-Corrected Charged AdS Black Hole
}

\author{
Meng-Sen Ma $\mathbb{D}^{1,2}$ and Yan-Song Liu ${ }^{2}$ \\ ${ }^{1}$ Institute of Theoretical Physics, Shanxi Datong University, Datong 037009, China \\ ${ }^{2}$ Department of Physics, Shanxi Datong University, Datong 037009, China
}

Correspondence should be addressed to Meng-Sen Ma; mengsenma@gmail.com

Received 2 August 2018; Revised 19 September 2018; Accepted 25 September 2018; Published 18 October 2018

Academic Editor: Rong-Gen Cai

Copyright (C) 2018 Meng-Sen Ma and Yan-Song Liu. This is an open access article distributed under the Creative Commons Attribution License, which permits unrestricted use, distribution, and reproduction in any medium, provided the original work is properly cited. The publication of this article was funded by SCOAP ${ }^{3}$.

\begin{abstract}
We study the thermodynamic properties and critical behaviors of the topological charged black hole in AdS space under the consideration of the generalized uncertainty principle (GUP). It is found that only in the spherical horizon case there are Van der Waals-like first-order phase transitions and reentrant phase transitions. From the equation of state we find that the GUP-corrected black hole can have one, two, and three apparent critical points under different conditions. However, it is verified by the Gibbs free energy that in either case there is at most one physical critical point.
\end{abstract}

\section{Introduction}

Since Hawking-Page phase transition of Schwarzschild-AdS black hole was explored in [1], phase structures and critical behaviors of various black holes in AdS space have been extensively studied [2-13]. Following [14, 15], the cosmological constant was considered as the thermodynamic pressure and the conjugated quantity was taken as the thermodynamic volume. In this extended phase space, the black hole mass $M$ should be identified with the enthalpy. Although in $[2,3]$, the Van der Waals (VdW)-like first-order phase transition was first found in the RN-AdS black hole, in the extended phase space it was found that the critical behaviors of the RN-AdS black hole have more similarities to that of the VdW liquid/gas system [16]. This finding aroused many relevant studies on the critical phenomena of various AdS black holes in the extended phase space [17-25]. Furthermore, some special critical behaviors such as the reentrant phase transition (RPT), the triple critical point, the isolated critical point, and even the "critical curve" for several black holes have been explored [26-35].

After considering quantum gravity effects, thermodynamic quantities of black holes may be modified. For example, the generalized uncertainty principle (GUP) will lead to the corrected temperature and entropy [36-43]. Thus, the GUP should also influence the critical behaviors of black holes correspondingly. In [44], the author studied the effects of the GUP to all orders in the Planck length on the thermodynamics and the phase transition of the Schwarzschild black hole. In this paper we consider the usually used simpler form of the GUP

$$
\Delta x \geq \frac{\hbar}{\Delta p}+\frac{\alpha^{2}}{\hbar} \Delta p \geq 2 \alpha \sim l_{p}
$$

where $l_{p}$ is the Planck length and $\alpha$ is a positive constant with length dimension whose upper limits can be given by the recent discovered gravitational waves [45]. On the basis of this relation, the corrected temperature and entropy for some static and stationary black holes were given in [46]. Using these corrected thermodynamic quantities, we have studied the critical behaviors of the Schwarzschild-AdS black hole and the RN-AdS black hole in [47]. With the GUP corrections, we find that the Hawking-Page phase transition for the AdS black holes no longer always occurs. In this paper, we will further study the critical behaviors and phase transitions of the corrected charged topological AdS black hole in the extended phase space. We find that a combination of $\alpha$ and the electric charge $Q$ can be used to classify the various kinds of critical behaviors.

The plan of this paper is as follows: In Section 2 we introduce the corrected thermodynamic quantities of the charged AdS black hole and simply discuss their properties. 
In Section 3 we find the critical points and analyze the numbers of the critical points. In Section 4 we study the critical behaviors of the black hole according to the Gibbs free energy. In Section 5 we summarize our results and discuss the possible future directions.

\section{Thermodynamics of the Charged Topological AdS Black Hole with GUP Correction}

In Einstein gravity in four-dimensional space-time, we have the charged topological AdS black hole solution,

$$
d s^{2}=-f(r) d t^{2}+f(r)^{-1} d r^{2}+r^{2} d \Omega_{k}^{2},
$$

with the metric function [48]

$$
f(r)=k-\frac{8 \pi G M}{\sum_{k} r}+\frac{16 \pi^{2} G^{2} Q^{2}}{\sum_{k}^{2} r^{2}}+\frac{r^{2}}{l^{2}},
$$

where the parameters $M, Q$ are the ADM mass and electric charge of the black hole and $l$ represents the cosmological radius. $d \Omega_{k}^{2}$ denotes the line element of a two-dimensional Einstein space with constant scalar curvature $2 k$ and volume $\Sigma_{k}$. Without loss of generality, one can take $k=1$ (spherical horizon), $k=0$ (planar/toroidal horizon), and $k=-1$ (hyperbolic horizon). Besides, we set $4 \pi G / \Sigma_{k}=1$ for simplicity. Although $\Sigma_{k}$ has different values for different $k$, this simplification will not affect our physical results.

According to the metric function in (3), the black hole mass is

$$
M=\frac{3 k r_{h}^{2}+8 \pi P r_{h}^{4}+3 Q^{2}}{6 r_{h}}
$$

where $r_{h}$ denotes the position of the event horizon of the black hole. Here $P$ is the thermodynamic pressure and is taken to be $P=-\Lambda / 8 \pi=3 / 8 \pi l^{2}$.

The surface gravity of the black hole is

$$
\kappa=\frac{f^{\prime}\left(r_{h}\right)}{2}=\frac{k r_{h}^{2}+8 \pi P r_{h}^{4}-Q^{2}}{2 r_{h}^{3}} .
$$

In the semiclassical case, the temperature and entropy for the black hole are

$$
\begin{aligned}
& T=\frac{\hbar \kappa}{2 \pi}, \\
& S=\frac{A}{4 \hbar} .
\end{aligned}
$$

As a thermodynamic system, the thermodynamic quantities of the black hole should satisfy the thermodynamic identity:

$$
d M=T d S+\Phi d Q+V d P
$$

where the electric potential measured at infinity with reference to the horizon is $\Phi=Q / r_{h}$ and the thermodynamic volume is $V=4 \pi r_{h}^{3} / 3$.
Generally, black hole entropy should be a function of the horizon area; namely, $S=S(A)$ [49]. Therefore, the temperature of a black hole can be generally expressed as [46]

$$
T=\left.\frac{\partial M}{\partial S}\right|_{Q}=\frac{d A}{d S} \times\left.\frac{\partial M}{\partial A}\right|_{Q}=\frac{d A}{d S} \times \frac{\kappa}{8 \pi} .
$$

According to Heisenberg uncertainty principle, one can derive $d A / d S \simeq \Delta A / \Delta S=$ const. This is just the work of Bekenstein and Hawking, which gives the results in (6).

Considering the effect of GUP, it is shown that [46]

$$
\frac{d A}{d S} \simeq \frac{(\Delta A)_{\min }}{(\Delta S)_{\min }}=4 \hbar^{\prime}
$$

where $\hbar^{\prime}$ is the effective Planck "constant" and is defined as

$$
\hbar^{\prime}=\frac{2 \hbar}{\alpha^{2}}\left(r_{h}^{2}-r_{h} \sqrt{r_{h}^{2}-\alpha^{2}}\right)
$$

Thus, the GUP-corrected black hole temperature becomes

$$
T^{\prime}=\frac{\hbar^{\prime} \kappa}{2 \pi}=\frac{\hbar\left(r_{h}-\sqrt{r_{h}^{2}-\alpha^{2}}\right)\left(k r_{h}^{2}+8 \pi P r_{h}^{4}-Q^{2}\right)}{2 \pi \alpha^{2} r_{h}^{2}} .
$$

From (5), one can see that the usual temperature of the charged AdS black hole will become negative for very small $r_{h}$, while $T^{\prime}$ give a mandatory requirement $r_{h} \geq \alpha$, from which we find that the temperature $T^{\prime}$ can be always positive when the condition $Q^{2}<\alpha^{2}\left(k+8 \pi P \alpha^{2}\right)$ is satisfied. In Figure 1, we compare the behaviors of the usual temperature $T$ and the corrected temperature $T^{\prime}$ for the charged AdS black hole. For smaller $Q, T^{\prime}$ is indeed always positive. Besides, with the GUP corrections the $T^{\prime}-r$ curve exhibits more fruitful structures.

Because GUP only constrains the minimal length, it only influences the temperature and the entropy. The electric charge and the electric potential will remain unchanged. The first law of black hole thermodynamics $d M=T^{\prime} d S^{\prime}+\Phi d Q+$ $V d P$ should still be established in this case. Therefore, the GUP-corrected entropy of the black hole can be derived.

$$
\begin{aligned}
& S^{\prime}=\left.\int \frac{d M}{T^{\prime}}\right|_{Q, P}=\left.\int \frac{1}{T^{\prime}} \frac{\partial M}{\partial r}\right|_{Q, P} d r+S_{0}=\frac{\pi}{2 \hbar}\left[r_{h}^{2}\right. \\
& \left.\quad+r_{h} \sqrt{r_{h}^{2}-\alpha^{2}}-\alpha^{2} \ln \left(\frac{\sqrt{r_{h}^{2}-\alpha^{2}}+r_{h}}{\alpha}\right)\right], \\
& =\frac{A}{4 \hbar}-\frac{\pi \alpha^{2}}{4 \hbar} \ln \frac{A}{\pi \alpha^{2}}+\cdots
\end{aligned}
$$

Here the effect of GUP leads to a subleading logarithmic term, which also exists in many other quantum corrected entropy. Our entropy is a little different from that in [46], where the authors take an indefinite integral and treat the integral constant as zero. We take the integration constant $S_{0}=$ $\alpha^{2} \ln \alpha$ to obtain a dimensionless logarithmic term. $S_{0}$ cannot be fixed by some physical consideration. To determine $S_{0}$ 

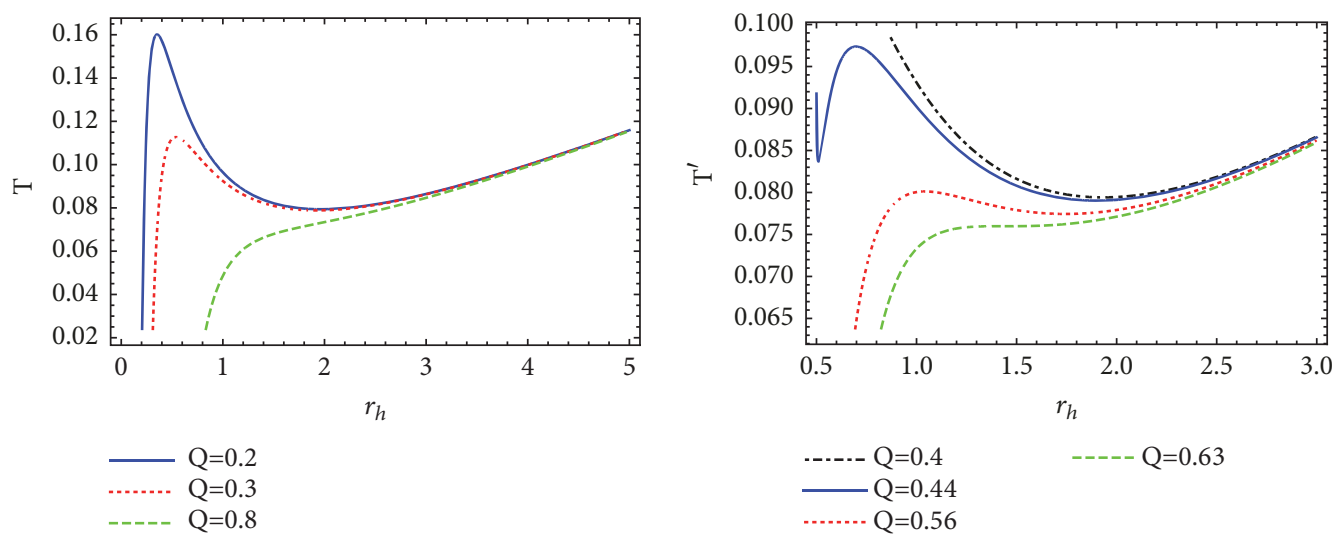

FIGURE 1: The left panel: the standard temperature of the RN-AdS black hole. The right panel: the GUP-corrected temperature with $\alpha=0.5$. In both cases we take $k=1$ and $P=0.01$.
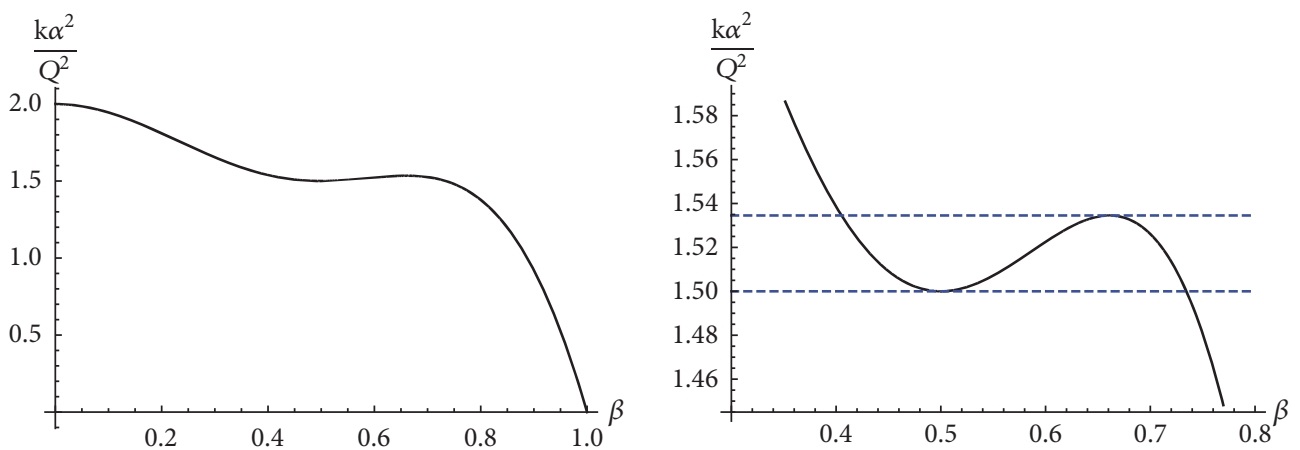

FiguRE 2: Number of the apparent critical points. There are at most three critical points for $1.5<\alpha^{2} / Q^{2}<1.535$.

completely, one has to invoke the quantum theory of gravity. It should be noted that the corrected entropy is independent of the parameter $k$ and it is always positive. Moreover, due to the existence of the logarithmic term in the corrected entropy, the Smarr formula no more exists.

\section{Multiple Critical Points}

In this section, we try to ascertain the number of the critical points. Below we always set $\hbar=1$ for simplicity. From (11), we can derive the equation of state

$$
P=\frac{r_{h}^{2}\left[2 \pi T^{\prime}\left(\sqrt{r_{h}^{2}-\alpha^{2}}+r_{h}\right)-k\right]+Q^{2}}{8 \pi r_{h}^{4}} .
$$

To derive the critical points, one should solve the following two equations:

$$
\frac{\partial P}{\partial r_{h}}=\frac{\partial^{2} P}{\partial r_{h}^{2}}=0
$$

One can also use another equivalent pair of equations, $\partial T^{\prime} / \partial r_{h}=\partial^{2} T^{\prime} / \partial r_{h}^{2}=0$, to determine the critical points of the system. In either case, the results are the same.
The two expressions are lengthy, so we will not list them here. Combining them, we obtain an equation

$$
\begin{aligned}
r_{h}^{4}( & \left.12 Q^{2}-\alpha^{2} k\right)+2 r_{h}^{3} \sqrt{r_{h}^{2}-\alpha^{2}}\left(\alpha^{2} k+3 Q^{2}\right) \\
& -3 k r_{h}^{5} \sqrt{r_{h}^{2}-\alpha^{2}}-26 \alpha^{2} Q^{2} r_{h}^{2} \\
& -4 \alpha^{2} Q^{2} r_{h} \sqrt{r_{h}^{2}-\alpha^{2}}+16 \alpha^{4} Q^{2}=0 .
\end{aligned}
$$

We set $\beta=\sqrt{1-\alpha^{2} / r_{h}^{2}}$; thus $0 \leq \beta \leq 1$. Utilizing $\beta$, (16) can be simplified to

$$
\begin{aligned}
& \alpha^{4}(2 \beta+1)\left[\alpha^{2}\left(\beta^{2}-\beta+1\right) k\right. \\
& \left.\quad+2\left(4 \beta^{5}-\beta^{4}-5 \beta^{3}+2 \beta^{2}+\beta-1\right) Q^{2}\right]=0 .
\end{aligned}
$$

In the case of $\alpha \neq 0$, we obtain a constraint equation

$$
\frac{k \alpha^{2}}{Q^{2}}=\frac{2\left(4 \beta^{5}-\beta^{4}-5 \beta^{3}+2 \beta^{2}+\beta-1\right)}{-\beta^{2}+\beta-1} .
$$

As is depicted in Figure 2, the right-hand side of (18) is nonnegative (in fact, when $\beta=1$, the RHS of (18) is zero; however, because we are only interested in the nontrivial case with $\alpha \neq 0$, this excludes the possibility of $\beta=1$ ). This means 


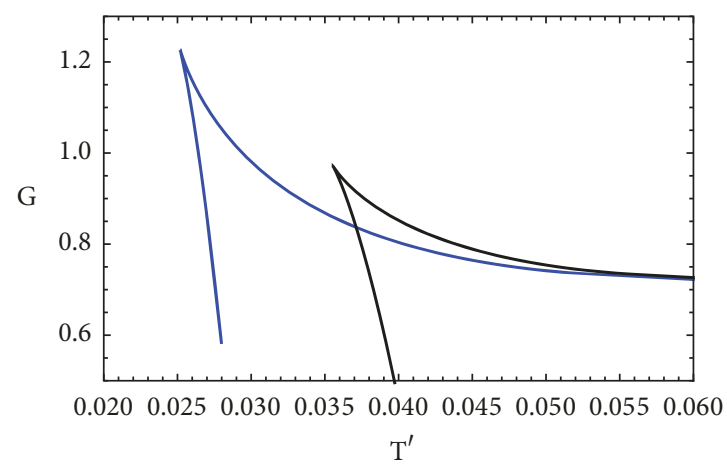

Figure 3: The case with $\alpha^{2} / Q^{2}=1.6$. The blue curve and the black curve correspond to $P=0.001$ and $P=0.002$, respectively.
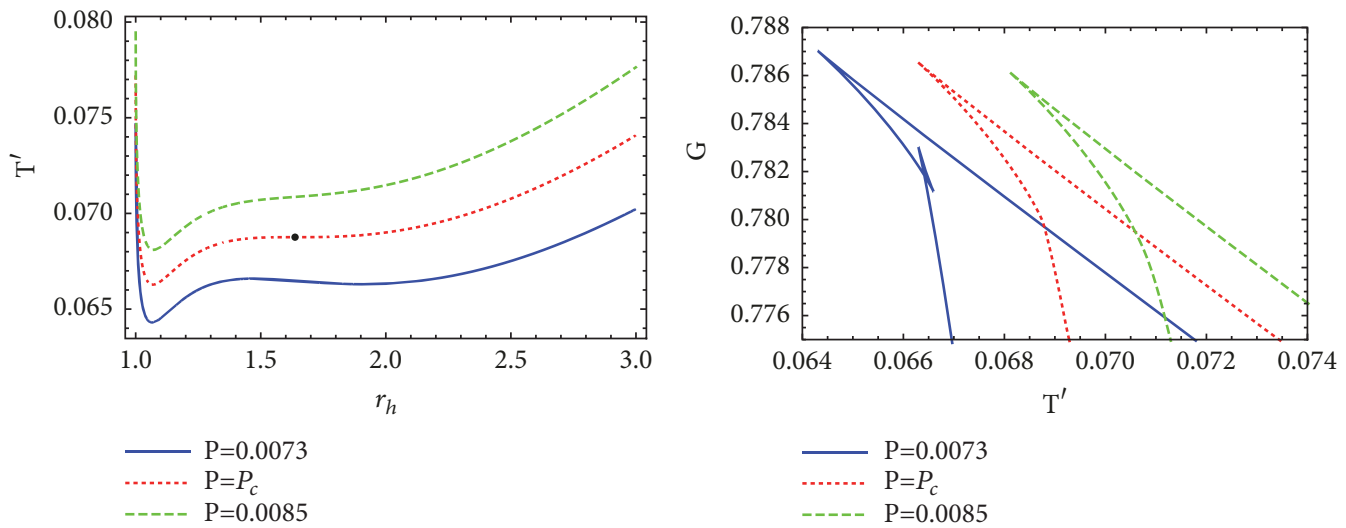

Figure 4: The $\alpha^{2} / Q^{2}=1.4$ case with $\alpha=1$ and $Q=0.845$. The critical pressure is $P_{c}=0.0079$.

that there is no $P-V$ criticality in the cases with $k=0$ and $k=-1$. Besides, when the electric charge $Q=0$, (17) becomes

$$
k \alpha^{6}(2 \beta+1)\left(\beta^{2}-\beta+1\right)=0,
$$

which also has no real solutions for $\beta$ in the range $0 \leq \beta \leq 1$. If $k=0$, this equation is well satisfied; however one can easily check that there is still no critical point in the $(k=0, Q=0)$ case.

Thus, below we are only concerned with the $k=1$ case with nonzero electric charge $Q$. We find that the number of critical points depends on the value of $\alpha^{2} / Q^{2}$. When $\alpha^{2} / Q^{2}>$ 1.535 or $\alpha^{2} / Q^{2}<1.5$, there is only one critical point. When $\alpha^{2} / Q^{2}=1.535$ or $\alpha^{2} / Q^{2}=1.5$, there are two critical points. And three critical points occur when $1.5<\alpha^{2} / Q^{2}<1.535$.

On the basis of these critical points, we can further discuss the heat capacity at constant pressure,

$$
C_{p}=\left.T^{\prime} \frac{\partial S^{\prime}}{\partial T^{\prime}}\right|_{P, Q}=\left.T^{\prime} \frac{\partial S^{\prime} / \partial r_{h}}{\partial T^{\prime} / \partial r_{h}}\right|_{P, Q},
$$

which can reflect the local thermodynamic stability of the black hole. The divergence points of the heat capacity occur at zeros of $\partial T^{\prime} / \partial r_{h}$, which are the extremal points in the $T^{\prime}-r_{h}$ curve. The sign of $C_{p}$ is also completely determined by $\partial T^{\prime} / \partial r_{h}$ because $\partial S^{\prime} / \partial r_{h}=\pi\left(\sqrt{r_{h}^{2}-\alpha^{2}}+r_{h}\right)>0$ and the corrected temperature is greater than zero if $Q^{2}<\alpha^{2}(1+8 \pi P)$.

\section{The Critical Behaviors and Gibbs Free Energy}

Below we discuss the critical behaviors of the RN-AdS black hole according to the numbers of the apparent critical points.

4.1. One Critical Point. In this case, we take $\alpha^{2} / Q^{2}=1.4$ and $\alpha^{2} / Q^{2}=1.6$, respectively. First, for $\alpha^{2} / Q^{2}=1.6$ one can easily find that the critical value of the pressure is negative, which means that no second-order phase transition occurs. In fact, there is also no VdW-like first-order phase transition. As is shown in Figure 3, the Gibbs free energy exhibits a cusp for any positive given pressure.

The critical behaviors in the case with $\alpha^{2} / Q^{2}=1.4$ have been analyzed in [47]. When the pressure is greater than the critical pressure $P_{c}$, there is two branches in the $T^{\prime}-r_{h}$ curve, which corresponds to a cusp in $G$. When the pressure is lower than $P_{c}$, the $T^{\prime}-r_{h}$ curve exhibits four branches. From left to right, we call them the small black hole, the left-intermediate black hole, the right-intermediate black hole, and the large black hole. According to the slope of the $T^{\prime}-r_{h}$ curve one can figure out that $C_{p}$ is negative in the small black hole branch and the right-intermediate black hole branch and it is positive in the left-intermediate black hole branch and the large black hole branch. From the $G-T^{\prime}$ figure, one can see a standard VdW-like first-order phase transition. We illustrate this in Figure 4. For $P \in\left(P_{t}, P_{z}\right)$, reentrant phase transition takes 

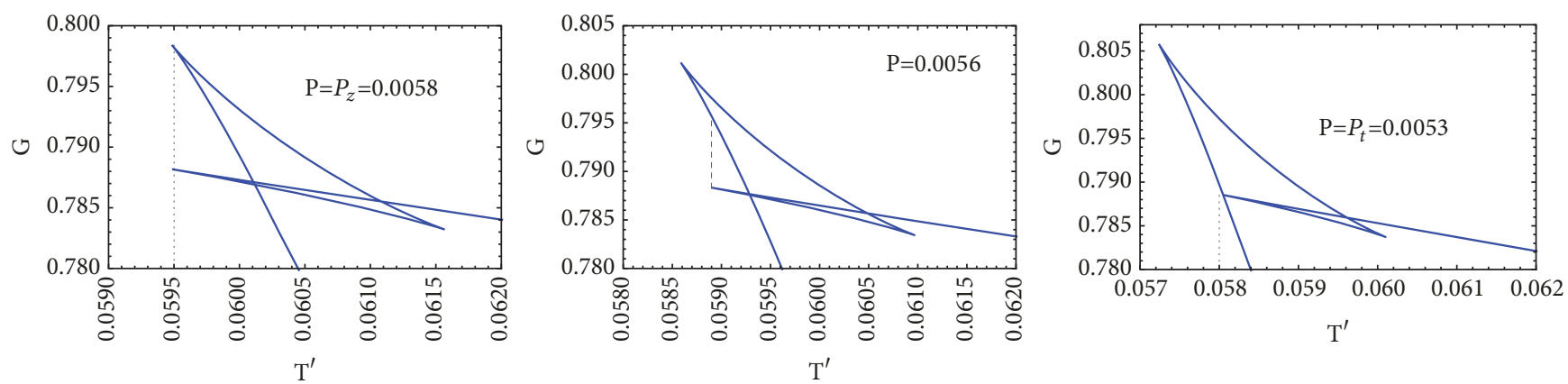

Figure 5: Characteristic behavior of Gibbs free energy for the reentrant phase transition. Here we also take $\alpha=1$ and $Q=0.845$.
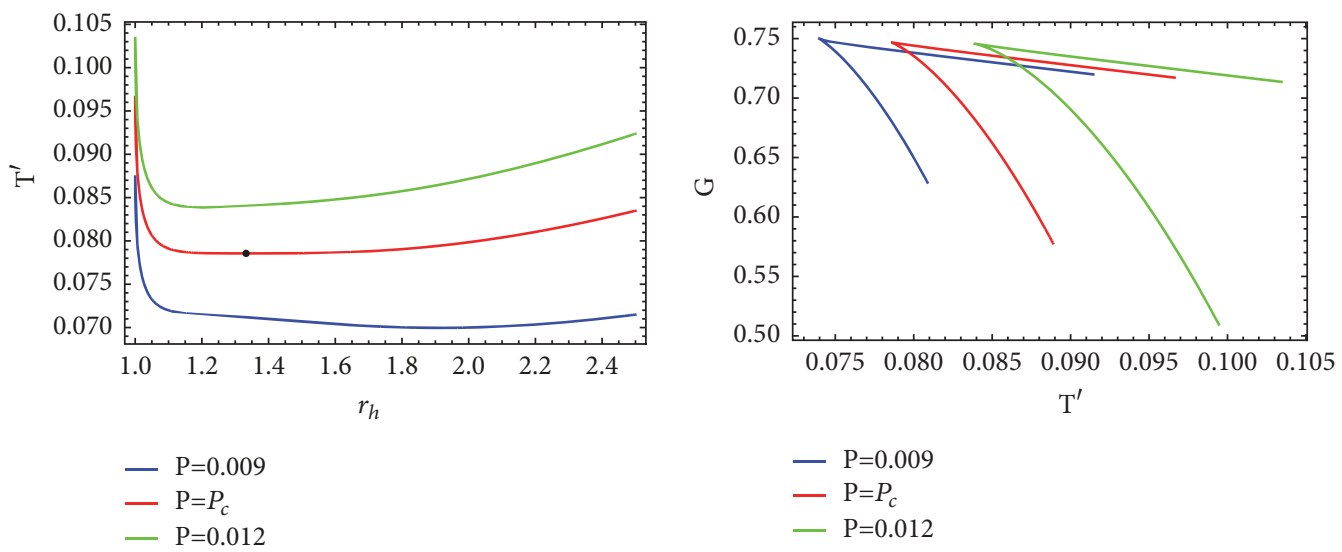

FIGURE 6: The $\alpha^{2} / Q^{2}=1.535$ case with $\alpha=1$ and $Q=0.807$.

place. In this case, starting off from the largest $G$, the black hole will first evolve along the branch of the large black hole. Then at some point the black hole will undergo a zero-order phase transition and jump to the left-intermediate branch. Finally, undergoing a first-order phase transition, the black hole returns back to the original large black hole. This process has been illustrated in Figure 5. When $P<P_{t}$, the large black hole is globally thermodynamic stable, and thus no phase transition occurs.

4.2. Two Critical Points. When $\alpha^{2} / Q^{2}=1.535$, one can easily check that the smaller critical point is false because the critical pressure $P_{c}$ is negative. The larger critical point is $\left(P_{c}=\right.$ $\left.0.0103, T_{c}=0.0786, r_{h c}=1.333\right)$. However, as is shown in Figure 6, the black hole only has two branches and exhibits a cusp in $G$. The lower branch has the positive heat capacity and smaller Gibbs free energy. Thus it is more stable for the large black hole and no phase transition occurs in this case.

For $\alpha^{2} / Q^{2}=1.5$, the smaller critical point is $\left(T_{c 1}=\right.$ 0.0613, $\left.P_{c 1}=0.00497, r_{c 1}=1.155\right)$, and the larger critical point is $\left(T_{c 2}=0.0753, P_{c 2}=0.00945, r_{c 2}=1.474\right)$. As is illustrated in Figure 7, for $P \in\left(P_{c 1}, P_{c 2}\right)$ the black hole has four branches. Similar to the one critical point case $\left(\alpha^{2} / Q^{2}=\right.$ 1.4), for $P \in\left(P_{t}, P_{c 2}\right)$ the black hole always has a VdWlike first-order phase transition and for $P \in\left(P_{t}, P_{z}\right)$ there exists the reentrant phase transition. Below a physical critical

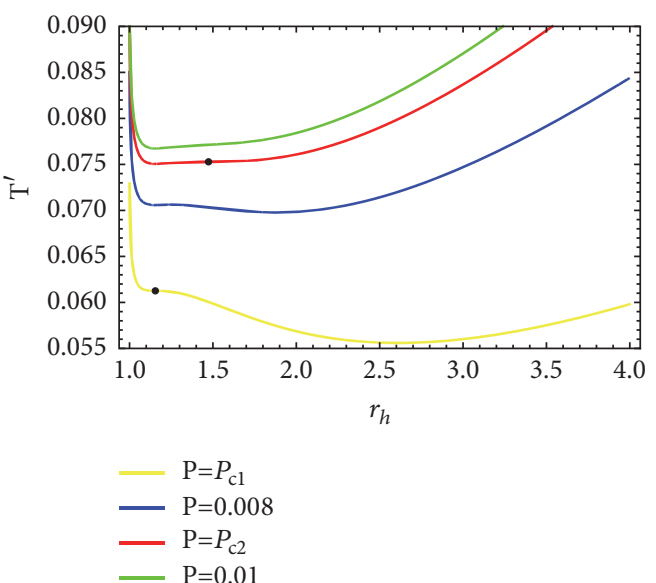

Figure 7: The $\alpha^{2} / Q^{2}=1.5$ case with $\alpha=1$ and $Q=0.816$. The reentrant phase transition takes place for $P \in\left(P_{t}, P_{z}\right)$ with $P_{t}=0.00887, P_{z}=0.009$.

point there should be a VdW-like first-order phase transition. Because $P_{c 1}<P_{t}$, for $P \in\left(P_{c 1}, P_{t}\right)$ or $P<P_{c 1}$ the large black hole is always globally thermodynamically stable. Therefore, the smaller critical point is indeed an apparent one, which does not correspond to any second-order phase transition. In this case, the Gibbs free energy has similar behaviors to that in Figures 4 and 5. 

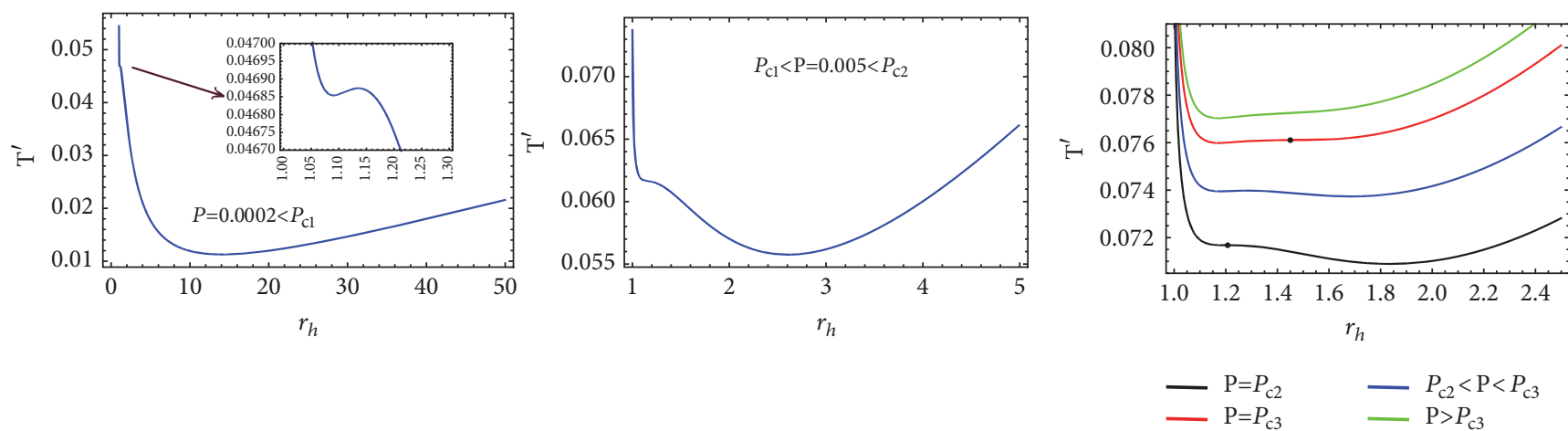

Figure 8: $T^{\prime}-r_{h}$ curves for $\alpha^{2} / Q^{2}=1.51$ with $\alpha=1$ and $Q=0.814$
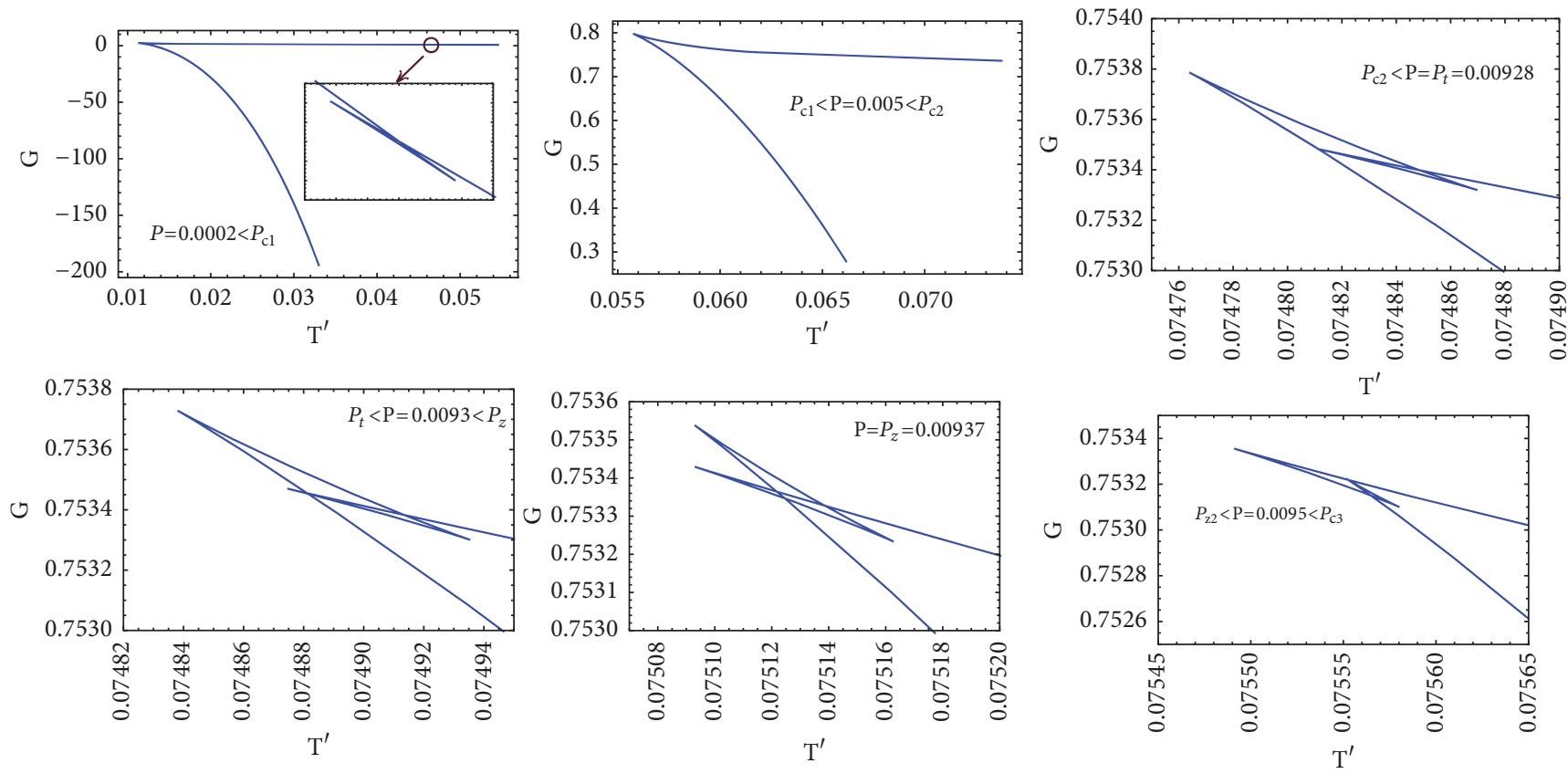

FIGURE 9: The different behaviors of the Gibbs free energy at different pressures. $\alpha^{2} / Q^{2}=1.51$ with $\alpha=1$ and $Q=0.814$.

4.3. Three Critical Points. In this case, we select $\alpha^{2} / Q^{2}=$ 1.51. Three apparent critical points are $\left(T_{c 1}=0.0497, P_{c 1}=\right.$ $\left.0.00111, r_{c 1}=1.118\right),\left(T_{c 2}=0.0717, P_{c 2}=0.00826, r_{c 2}=\right.$ $1.207)$, and $\left(T_{c 3}=0.0761, P_{c 3}=0.00966, r_{c 3}=1.45\right)$, respectively.

As is shown in Figure 8, for $P<P_{c 1}$ and $P \in\left(P_{c 2}, P_{c 3}\right)$, there are four branches and there are two branches for $P>P_{c 3}$ and $P \in\left(P_{c 1}, P_{c 2}\right)$. According to the illustration in Figure 9 , when $P<P_{c 1}$ and $P_{c 2}<P<P_{t}$, there are swallowtail behaviors; however the swallowtail never intersects with the large black hole branch. When $P \in\left(P_{c 1}, P_{c 2}\right)$, it is a cusp in the Gibbs free energy. This means that the large black hole is always globally thermodynamically stable when $P<P_{t}$ and " $c 1$ " and " $c 2$ " are not physical critical points. For $P>P_{c 3}$, the Gibbs free energy also exhibits a cusp and for $P \in\left(P_{t}, P_{c 3}\right)$ there is always a phase transition of first order. Thus, " $c 3$ " is a physical critical point, which corresponds to a second-order phase transition.

\section{Conclusion and Discussion}

We begin by considering the charged AdS black hole with general topology $(k=0, \pm 1)$. After considering the GUP we find that the temperature of the RN-AdS black hole, not like its semiclassical counterpart, can be always positive and has more fruitful structures. The GUP-corrected entropy is always positive and is independent of the electric charge $Q$ and the parameter $k$. By analyzing the equation of state, we find that only in the $k=1$ case the black hole can have critical behaviors. In particular, one can judge the number of the critical points according to the values of $\alpha^{2} / Q^{2}$. Apparently, the number of the critical points can be one, two, and three. In either case, there is the unique physical critical point. The main results have been summarized in Table 1.

For $P>1.535$, the apparent critical point has a negative pressure, which is unphysical. At any positive pressure, the Gibbs free energy exhibits a cusp. For $P=1.535$, the smaller 
TABLE 1: The critical behaviors of RN-AdS black hole with GUP corrections in the $k=1$ case.

\begin{tabular}{lccr}
\hline$\alpha^{2} / Q^{2}$ & \# apparent critical points & \# physical critical points & behavior \\
\hline$\left(\frac{1}{1+8 \pi P}, 1.5\right)$ & 1 & 1 & VdW\& RPT \\
1.5 & 2 & 1 & VdW\& RPT \\
$(1.5,1.535)$ & 3 & 1 & VdW\& RPT \\
1.535 & 2 & 0 & cusp \\
$(1.535, \infty)$ & 1 & 0 & cusp \\
\hline
\end{tabular}

one of the two apparent critical points also has negative pressure. Below or above the larger critical point, the $T^{\prime}-r_{h}$ curve only has two branches, which corresponds to a cusp in the Gibbs free energy. For $1.5 \leq P<1.535$, only the rightmost critical point is the physical one. In the first three cases in Table 1, there is always the VdW-like phase transitions, which occur at the range $P \in\left(P_{t}, P_{c}\right), P \in\left(P_{t}, P_{c 2}\right), P \in\left(P_{t}, P_{c 3}\right)$, respectively, and the RPT takes place for $P \in\left(P_{t}, P_{z}\right)$.

The Born-Infeld-AdS black hole and the Kerr-AdS black hole can have the reentrant behavior due to their complex horizon structure. GUP only modifies the thermodynamic quantities, but not the geometric structure like the metric. If we take into account the corrections of the GUP in these complicated black holes, there should be more interesting phase structure and critical phenomena. This will be left for future studies.

\section{Data Availability}

The data used to support the findings of this study are available from the corresponding author upon request.

\section{Conflicts of Interest}

The authors declare that they have no conflicts of interest.

\section{Acknowledgments}

This work is supported in part by the National Natural Science Foundation of China (Grants No. 11605107 and No. 11475108 ) and by the Natural Science Foundation of Shanxi (Grant No. 201601D021022).

\section{References}

[1] S. W. Hawking and D. N. Page, "Thermodynamics of black holes in anti-de Sitter space," Communications in Mathematical Physics, vol. 87, no. 4, pp. 577-588, 1982/83.

[2] A. Chamblin, R. Emparan, C. V. Johnson, and R. C. Myers, "Charged AdS black holes and catastrophic holography," Physical Review D: Particles, Fields, Gravitation and Cosmology, vol. 60, no. 6, 1999.

[3] A. Chamblin, R. Emparan, C. V. Johnson, and R. C. Myers, "Holography, thermodynamics, and fluctuations of charged AdS black holes," Physical Review D: Particles, Fields, Gravitation and Cosmology, vol. 60, no. 10, Article ID 104026, 1999.

[4] C. S. Peça and J. P. S. Lemos, "Thermodynamics of ReissnerNordström-anti-de Sitter black holes in the grand canonical ensemble," Physical Review D: Particles, Fields, Gravitation and Cosmology, vol. 59, no. 12, Article ID 124007, 1999.

[5] X. N. Wu, "Multicritical phenomena of Reissner-Nordström anti-de Sitter black holes," Physical Review D: Particles, Fields, Gravitation and Cosmology, vol. 62, no. 12, Article ID 124023, 2000.

[6] Y. S. Myung, Y.-W. Kim, and Y.-J. Park, "Thermodynamics and phase transitions in the Born-Infeld-anti-de Sitter black holes," Physical Review D: Particles, Fields, Gravitation and Cosmology, vol. 78, no. 8, Article ID 084002, 2008.

[7] H. Quevedo and A. Sanchez, "Geometrothermodynamics of asymptotically anti-de Sitter black holes," Journal of High Energy Physics, no. 9, 034 pages, 2008.

[8] M. Cadoni, G. D’Appollonio, and P. Pani, "Phase transitions between Reissner-Nordstrom and dilatonic black holes in 4D AdS spacetime," Journal of High Energy Physics, no. 3, 100, 27 pages, 2010.

[9] H. Liu, H. Lü, M. Luoa, and K.-N. Shao, "Thermodynamical metrics and black hole phase transitions," Journal of High Energy Physics, vol. 2010, no. 12, article 054, 2010.

[10] A. Sahay, T. Sarkar, and G. Sengupta, "Thermodynamic geometry and phase transitions in Kerr-Newman-AdS black holes," Journal of High Energy Physics, vol. 2010, article 118, 2010.

[11] R. Banerjee, S. K. Modak, and S. Samanta, "Second order phase transition and thermodynamic geometry in Kerr-AdS black holes," Physical Review D: Particles, Fields, Gravitation and Cosmology, vol. 84, Article ID 064024, 2011.

[12] M.-S. Ma, F. Liu, and R. Zhao, "Continuous phase transition and critical behaviors of 3D black hole with torsion," Classical and Quantum Gravity, vol. 31, no. 9, Article ID 095001, 2014.

[13] M.-S. Ma and R. Zhao, "Phase transition and entropy spectrum of the BTZ black hole with torsion," Physical Review D: Particles, Fields, Gravitation and Cosmology, vol. 89, no. 4, Article ID 044005, 2014.

[14] D. Kastor, S. Ray, and J. Traschen, "Enthalpy and the mechanics of AdS black holes," Classical and Quantum Gravity, vol. 26, no. 19, Article ID 195011, 16 pages, 2009.

[15] B. P. Dolan, "Pressure and volume in the first law of black hole thermodynamics," Classical and Quantum Gravity, vol. 28, no. 23, Article ID 235017, 2011.

[16] D. Kubiznak and R. B. Mann, "P-V criticality of charged AdS black holes," Journal of High Energy Physics, vol. 2012, no. 7, article 033, 2012.

[17] R.-G. Cai, L.-M. Cao, L. Li, and R.-Q. Yang, "P-V criticality in the extended phase space of Gauss-Bonnet black holes in AdS space," Journal of High Energy Physics, vol. 2013, no. 9, article 5, 2013.

[18] S. Chen, X. Liu, and C. Liu, "P-V Criticality of an AdS Black Hole in $\mathrm{f}(\mathrm{R})$ Gravity," Chinese Physics Letters, vol. 30, no. 6, Article ID 060401, 2013. 
[19] S. H. Hendi and M. H. Vahidinia, "Extended phase space thermodynamics and P-V criticality of black holes with a nonlinear source," Physical Review D: Particles, Fields, Gravitation and Cosmology, vol. 88, no. 8, Article ID 084045, 11 pages, 2013.

[20] N. Altamirano, D. Kubizňák, R. Mann, and Z. Sherkatghanad, "Thermodynamics of rotating black holes and black rings: phase transitions and thermodynamic volume," Galaxies, vol. 2, no. 1, pp. 89-159, 2014.

[21] J.-X. Mo and W.-B. Liu, " $P-V$ criticality of topological black holes in Lovelock-Born-Infeld gravity," The European Physical Journal C, vol. 74, article 2836, 2014.

[22] H. Xu, W. Xu, and L. Zhao, "Extended phase space thermodynamics for third-order Lovelock black holes in diverse dimensions," The European Physical Journal C, vol. 74, article 3074, 2014.

[23] M.-S. Ma and Y.-Q. Ma, "Critical behaviors of a black hole in an asymptotically safe gravity with cosmological constant," Classical and Quantum Gravity, vol. 32, no. 3, p. 035024, 2015.

[24] M.-S. Ma and R. Zhao, "Stability of black holes based on horizon thermodynamics," Physics Letters B, vol. 751, pp. 278-283, 2015.

[25] H.-H. Zhao, L.-C. Zhang, M.-S. Ma, and R. Zhao, "Phase transition and Clapeyron equation of black holes in higher dimensional AdS spacetime," Classical and Quantum Gravity, vol. 32, no. 14, Article ID 145007, 2015.

[26] N. Altamirano, D. Kubiznak, and R. Mann, "Reentrant phase transitions in rotating AdS black holes," Physical Review D: Particles, Fields, Gravitation and Cosmology, vol. 88, no. 10, Article ID 101502, 5 pages, 2013.

[27] S.-W. Wei and Y.-X. Liu, "Triple points and phase diagrams in the extended phase space of charged Gauss-Bonnet black holes in AdS space," Physical Review D: Particles, Fields, Gravitation and Cosmology, vol. 90, no. 4, Article ID 044057, 2014.

[28] B. P. Dolan, A. Kostouki, D. Kubizňák, and R. B. Mann, "Isolated critical point from Lovelock gravity," Classical and Quantum Gravity, vol. 31, no. 24, Article ID 242001, 2014.

[29] A. M. Frassino, D. Kubizňák, R. B. Mann, and F. Simovic, "Multiple reentrant phase transitions and triple points in Lovelock thermodynamics," Journal of High Energy Physics, vol. 2014, no. 9, article 80, 2014.

[30] M. Zhang, D.-C. Zou, and R.-H. Yue, "Reentrant Phase Transitions and Triple Points of Topological AdS Black Holes in BornInfeld-Massive Gravity," Advances in High Energy Physics, vol. 2017, Article ID 3819246, 11 pages, 2017.

[31] A. Dehyadegari and A. Sheykhi, "Reentrant phase transition of Born-Infeld-AdS black holes," Physical Review D: Particles, Fields, Gravitation and Cosmology, vol. 98, no. 2, 2018.

[32] R. A. Hennigar, R. B. Mann, and E. Tjoa, "Superfluid black holes," Physical Review Letters, vol. 118, no. 2, Article ID 021301, 2017.

[33] R. A. Hennigar, E. Tjoa, and R. B. Mann, “Thermodynamics of hairy black holes in Lovelock gravity," Journal of High Energy Physics, vol. 2017, no. 2, article 70, 2017.

[34] H. Dykaar, R. A. Hennigar, and R. B. Mann, "Hairy black holes in cubic quasi-topological gravity," Journal of High Energy Physics, no. 5, 045, front matter+29 pages, 2017.

[35] M.-S. Ma and R.-H. Wang, "Peculiar P-V criticality of topological Hořava-Lifshitz black holes," Physical Review D: Particles, Fields, Gravitation and Cosmology, vol. 96, no. 2, Article ID 024052, 2017.

[36] R. J. Adler, P. Chen, and D. I. Santiago, "The generalized uncertainty principle and black hole remnants," General Relativity and Gravitation, vol. 33, no. 12, pp. 2101-2108, 2001.
[37] A. J. Medved and E. C. Vagenas, "When conceptual worlds collide: the generalized uncertainty principle and the BekensteinHawking entropy," Physical Review D: Particles, Fields, Gravitation and Cosmology, vol. 70, no. 12, Article ID 124021, 5 pages, 2004.

[38] Z. Ren and Z. Sheng-Li, "Generalized uncertainty principle and black hole entropy," Physics Letters. B. Particle Physics, Nuclear Physics and Cosmology, vol. 641, no. 2, pp. 208-211, 2006.

[39] K. Nouicer, "Quantum-corrected black hole thermodynamics to all orders in the Planck length," Physics Letters B, vol. 646, no. 2-3, pp. 63-71, 2007.

[40] W. Kim, E. J. Son, and M. Yoon, "Thermodynamics of a black hole based on a generalized uncertainty principle," Journal of High Energy Physics, vol. 2008, no. 1, article 35, 2008.

[41] B. Majumder, "Quantum black hole and the modified uncertainty principle," Physics Letters B, vol. 701, no. 4, pp. 384-387, 2011.

[42] Z. W. Feng, H. L. Li, X. T. Zu, and S. Z. Yang, "Quantum corrections to the thermodynamics of Schwarzschild-Tangherlini black hole and the generalized uncertainty principle," The European Physical Journal C, vol. 76, article 212, 2016.

[43] E. C. Vagenas, S. M. Alsaleh, and A. F. Ali, "GUP parameter and black-hole temperature," EPL (Europhysics Letters), vol. 120, no. 4, Article ID 40001, 2017.

[44] Y. Sabri and K. Nouicer, "Phase transitions of a GUP-corrected Schwarzschild black hole within isothermal cavities," Classical and Quantum Gravity, vol. 29, no. 21, 215015, 15 pages, 2012.

[45] Z.-W. Feng, S.-Z. Yang, H.-L. Li, and X.-T. Zu, "Constraining the generalized uncertainty principle with the gravitational wave event GW150914," Physics Letters B, vol. 768, pp. 81-85, 2017.

[46] L. Xiang and X. Q. Wen, "Black hole thermodynamics with generalized uncertainty principle," Journal of High Energy Physics, vol. 2009, no. 10, article 46, 2009.

[47] Z. Sun and M.-S. Ma, "The critical behaviors of the black holes with the generalized uncertainty principle," EPL (Europhysics Letters), vol. 122, no. 6, p. 60002, 2018.

[48] R.-G. Cai and K.-S. Soh, "Topological black holes in the dimensionally continued gravity," Physical Review D: Particles, Fields, Gravitation and Cosmology, vol. 59, no. 4, Article ID 044013, 1999.

[49] J. D. Bekenstein, "Black holes and entropy," Physical Review D: Particles, Fields, Gravitation and Cosmology, vol. 7, pp. 23332346, 1973. 

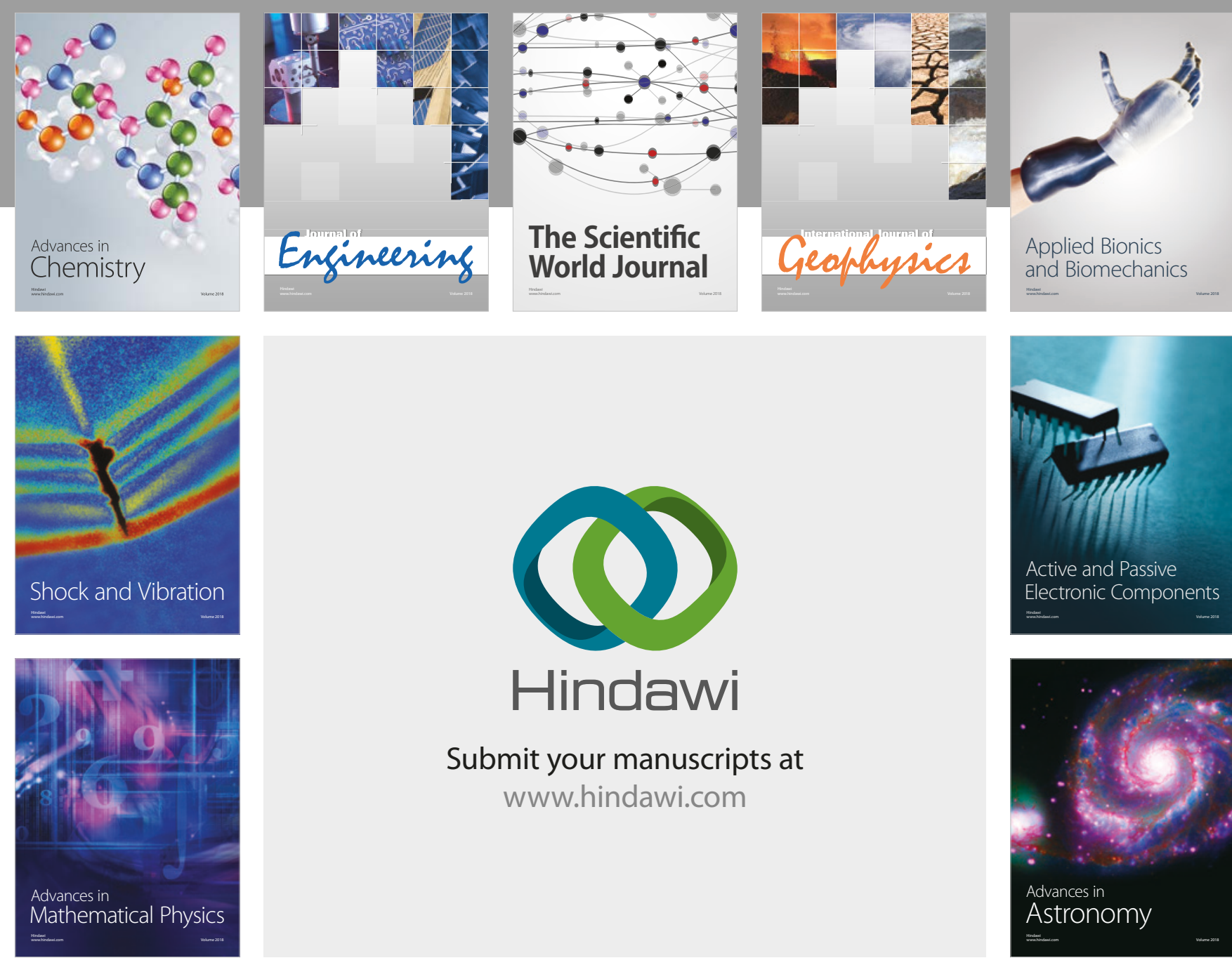

Submit your manuscripts at

www.hindawi.com

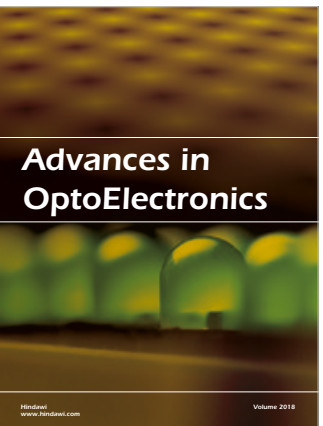

\section{Rotcting Machinery}
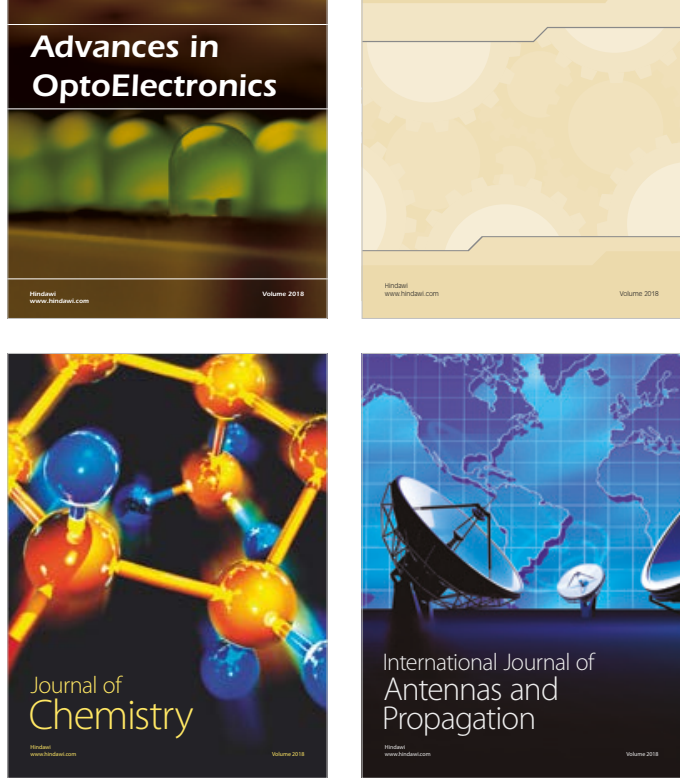

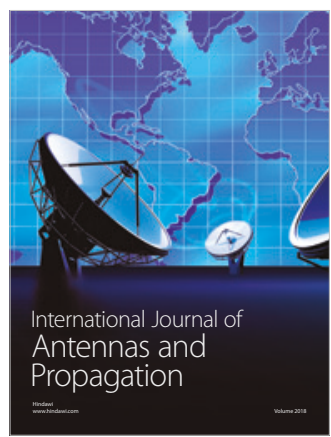

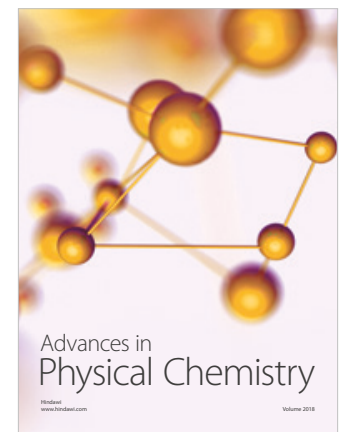

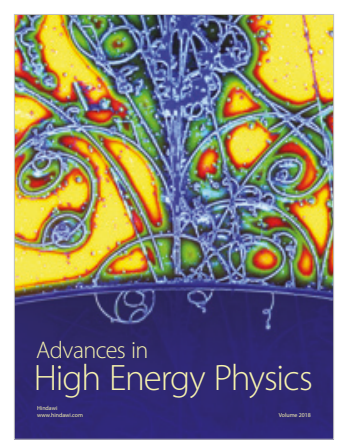

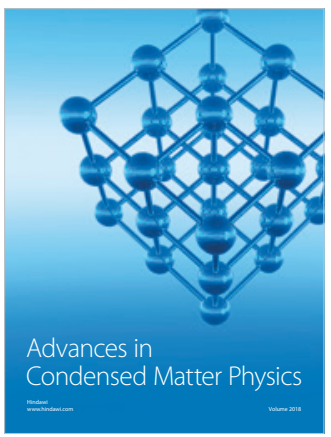

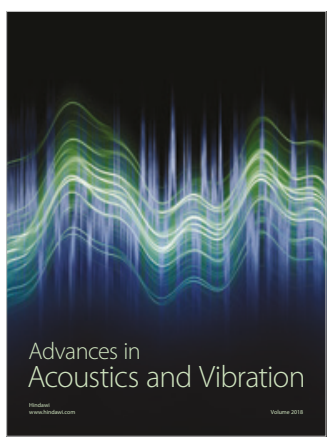

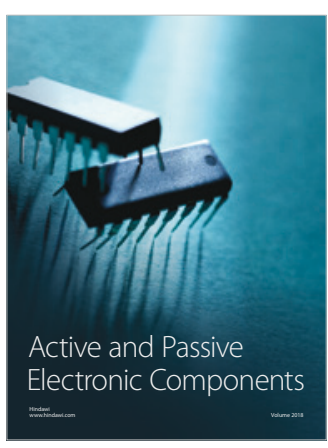
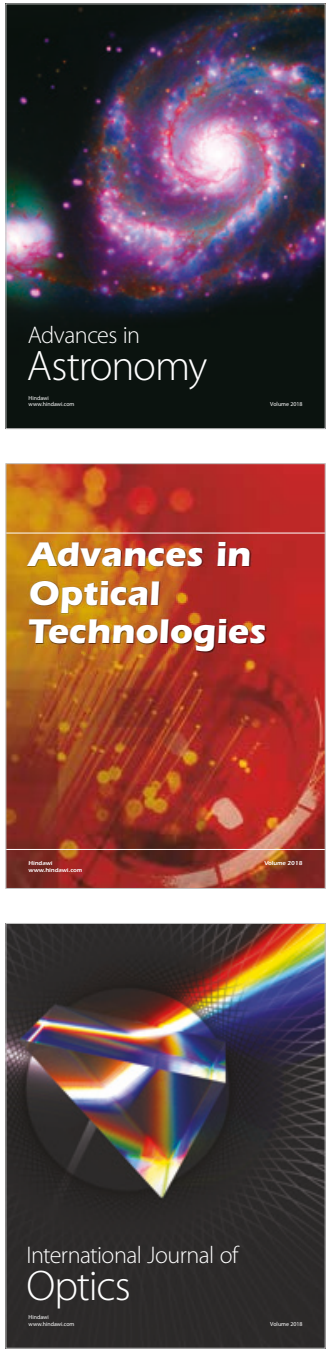